\title{
A colaboração científica na pesquisa sobre coautoria: um método baseado na análise de redes
}

Ricardo Barros Sampaio

Doutor - Faculdade de Ciência da Informação de Brasília.Grupo de Pesquisa em Inteligência Cooperativa em Redes Sociais ComplexaPesquisador - Fundação Oswaldo Cruz - DIREB

Helena Célia de Souza Sacerdote

Doutoranda - Faculdade de Ciência da

Informação - Universidade de Brasília

Bruna de Paula Fonseca Fonseca

Doutora - Engenharia de Produção Universidade Federal do Rio de Janeiro (UFRJ).Pesquisadora - Fundação Oswaldo Cruz - CDTS

Jorge Henrique Cabral Fernandes

Doutor - Professor no Programa de Pós Graduação da Faculdade de Ciência da Informação - Universidade de Brasília Grupo de Pesquisa em Inteligência Cooperativa em Redes Sociais Complexas

http://dx.doi.org/10.1590/1981-5344/2447

O presente trabalho apresenta um metodologia para o estudo da comunicação científica derivado dos métodos de análise de redes. São analisadas as relações profissionais de colaboração científica entre autores que utilizam a coautoria, uma nova subárea da comunicação científica que vem ganhando espaço na ciência da informação. Na coautoria, dois autores quaisquer são definidos como tendo um relacionamento caso tenham colaborado na produção de um artigo ou publicação científica. O artigo investiga se tal associação permite entender as problemáticas da colaboração científica, como por exemplo, se o conhecimento está concentrado em alguns poucos países ou indivíduos, e como esses 
indivíduos se relacionam. A metodologia desenvolvida se baseia no modelo exploratório proposto por Nooy, Mrvar e Batagelj (2011). Do ponto de vista da bibliometria, foram analisadas as colaborações científicas na base de dados Web of Science. Os resultados alcançados demonstraram que a pesquisa sobre coautoria se espalha por diversos países e por diferentes áreas de interesse. Observa-se que a associação dos métodos de bibliometria e análise de redes contribui para uma melhor interpretação de resultados, principalmente por meio da inspeção visual que a análise de redes proporciona.

Palavras Chave: Coautoria; Colaboração científica; Análise de redes.

\section{Scientific collaboration in co- authorship research: a method based on network analysis}

This work presents a method to the study of scientific communication derived from network analysis methods. It has been analyzed the professional relationships of scientific collaboration between authors that use coauthoship, a new sub-area in scientific communication that has gained space in information science. In coauthorship, any two authors are defined as having a relationship if they have collaborated in the production of an article or scientific publication. This paper investigates whether this association allows the comprehension of the problems of scientific collaboration, for example, if knowledge is concentrated in a few countries or individuals, and how these individuals relate to each other. The methodology is based on the exploratory model proposed by Nooy, Mrvar e Batagelj (2011). From the point of view of bibliometrics, scientific collaborations were analyzed in the Web of Science database. The results obtained showed that the field of co-authorship spans several countries and different areas of interest. It is observed that the association of bibliometric methods and network analysis contributes to a better interpretation of the results, mainly through the visual inspection that network analysis methods are able to provide. 
Key words: Co-authorship; Scientific collaboration; Network analysis.

Recebido em 26.05.2015 Aceito em 03.11.2015

\section{Introdução}

As redes de coautoria são um poderoso instrumento para a análise de colaborações e parcerias científicas e tecnológicas, proporcionando uma visão dos padrões de cooperação entre indivíduos e organizações (MELIN; PERSSON, 1996; NEWMAN, 2004; GLÄNZEL, 2002). A coautoria de um documento representa uma relação oficial do envolvimento de dois ou mais autores ou organizações (GLÄNZEL; SCHUBERT, 2004), e apesar do debate antigo a respeito do seu significado e interpretação (KATZ; MARTIN, 1997; LAUDEL, 2002), a análise de coautoria tem sido amplamente utilizada para entender e avaliar os padrões de colaboração científica.

A colaboração científica pode, então, ser definida como a interação que ocorre em um contexto social entre dois ou mais pesquisadores, que facilita o compartilhamento de significado e a realização de tarefas com relação a um objetivo mutuamente compartilhado (SONNENWALD, 2007).

Em redes de coautoria, a colaboração é retratada de tal maneira que os vértices representam os autores ou as organizações às quais esses estão vinculados, e dois ou mais autores estão conectados se eles compartilham a autoria de uma publicação (NEWMAN, 2004). Em uma perspectiva interorganizacional, a cooperação pode ser definida em termos de publicações que tenham autoria compartilhada por indivíduos afiliados a duas ou mais organizações (NAGPAUL, 2002).

Estudos empíricos apontam que tais redes de colaboração tendem a apresentar uma distribuição de ligações extremamente desigual e hierárquica (BARABÁSI et al., 2002; NEWMAN, 2004; GOYAL; LEIJ; MORAGA GONZÁLEZ, 2006), com grande concentração da produção científica em alguns poucos autores.

A ciência de redes é uma área do conhecimento que tem como foco o estudo de redes complexas, tais como redes de telecomunicação, computadores, biológicas, sociais, cognitivas, semânticas, entre outras. Ela se vale de teorias e métodos de áreas como matemática, estatística, física, ciência da computação, sociologia e outras (NATIONAL RESEARCH COUNCIL, 2005; NEWMAN, 2010). Ao longo da primeira década do século 21, houve uma explosão científica sobre a ciência das redes, fundamentalmente embasada na descoberta de que, apesar das diferenças aparentes, o surgimento e a evolução das diferentes redes é impulsionado por um conjunto comum de leis e mecanismos. Por esse motivo, apesar da incrível diversidade de forma, dimensão e natureza que caracterizam as redes reais, a maioria daquelas observadas na natureza, na sociedade e na tecnologia são movidas por princípios de organização comuns (BARABASI, 2012). É essa característica que dá à ciência das 
redes uma perspectiva multidisciplinar, que oferece novas ferramentas para uma ampla gama de áreas da pesquisa científica.

A Análise de Redes Sociais, aqui denominada apenas como análise de redes, é uma abordagem da ciência das redes oriunda de três campos teóricos: a Sociologia, a Antropologia e a Psicologia. Seu conceito é definido por Wasserman e Faust (1994) como um extenso conjunto de métodos para avaliação da estrutura de grupos sociais. Esses grupos podem formar redes sociais que consistem de um ou mais conjuntos finitos de atores, aqui denominados como vértices, e das relações estabelecidas entre eles. Sua principal característica é a ênfase não nos atributos (características) dos atores, mas nas ligações entre eles; ou seja, a unidade de observação é composta pelo conjunto de atores e suas conexões (WASSERMAN, FAUST, 1994).

O estudo de redes é aplicado em diversas disciplinas como meio de análise de dados relacionais complexos, diferenciando-se do estudo estatístico clássico, no qual o elemento de estudo e suas características são o principal objeto. A análise de redes, por sua vez, é a aplicação prática dos métodos estudados e descritos na ciência de redes.

O objetivo desse trabalho é avaliar a capacidade de análise de resultados bibliométricos associados aos métodos de análise de redes, os quais se baseiam na ciência de redes. A metodologia utilizada baseou-se no modelo exploratório proposto por Nooy, Mrvar e Batagelj (2011).

Uma das aplicações práticas da ciência de redes é o apoio na revisão bibliográfica por meio da análise de colaborações científicas. Buscou-se realizar a aplicação do método de análise de redes de colaboração científica ao universo de pesquisa sobre redes de coautoria com o intuito de entender se essa área está concentrada em alguns poucos países ou indivíduos e como esses indivíduos se relacionam.

Este artigo é composto por mais seis seções. A seção 2 apresenta a metodologia utilizada e tem como principal objetivo demonstrar os passos necessários para a análise, dentro do conceito de análise de redes. A seção 3 apresenta os dados bibliométricos referentes ao universo de pesquisa avaliado. O foco se deu na base Web of Science (WoS) e nas áreas de conhecimento relacionadas à Ciência da Informação. A seção 4 apresenta as redes, a descrição de suas características estruturais e sua inspeção visual. Por fim, a seção 5 apresenta a conclusão do trabalho.

\section{Metodologia}

Este estudo utilizou como referência o modelo exploratório de análise de redes proposto por Nooy, Mrvar e Batagelj (2011), na qual são definidas quatro etapas cíclicas, sendo elas: a definição da rede; 0 tratamento de dados da rede; a determinação de características estruturais; e a inspeção visual. Nooy, Mrvar e Batagelj (2011) descrevem que o principal objetivo da análise de redes é a detecção e a interpretação de padrões sociais nas relações entre os atores da rede. O foco do modelo e do estudo realizado neste artigo está nas redes sociais existentes na 
pesquisa sobre coautoria, onde os pesquisadores identificados nas publicações científicas são os atores ou vértices das redes.

O modelo proposto se diz exploratório por não haver hipótese específica acerca das características da estrutura da rede. Apesar de já existirem diversos estudos a respeito de redes de colaboração científica, os resultados específicos são dependentes do universo que está sendo estudado, seja ele temporal, por área ou qualquer outro fator.

\subsection{Definição da Rede}

Quanto à estrutura da rede, um grafo pode ser direcionado ou não, dependendo se a linha que une dois vértices carrega uma informação unilateral ou bidirecional. Um exemplo desses diferentes tipos de direcionalidade na pesquisa científica pode ser uma rede de citações e uma rede de coautoria. No primeiro caso o grafo é direcionado, pois, quando um pesquisador cita outro autor a recíproca, em muitos casos, não é verdadeira. No caso de um grafo de coautoria, este pode ser considerado não direcionado, pois se pressupõe a colaboração entre ambas as partes.

Outro elemento importante é a permissão de linhas múltiplas. No caso da rede de coautoria, são permitidas as linhas múltiplas, uma vez que um pesquisador pode colaborar diversas vezes com um mesmo companheiro. Isso pode ser demonstrado por meio da espessura (peso) das linhas que unem os pesquisadores. Quanto mais espessa a linha, maior é o número de colaborações entre os vértices. Outro elemento existente nos grafos, mas que não é considerado neste estudo, são os loops, ou seja, quando um vértice se relaciona consigo mesmo. No caso da colaboração por meio de coautoria isso não é considerado, mas, em uma rede de citação esse elemento pode ser analisado.

Uma vez que se tem o grafo e seus elementos, o próximo passo é a definição da rede, que nada mais é do que o grafo com informações adicionais acerca dos vértices e das linhas. Dessa maneira, quando se definem os nomes, os países, os grupos ou o peso das linhas na rede de pesquisa científica estão sendo definidos o contexto e a estrutura informacional da rede.

As redes analisadas neste estudo, portanto, são compostas de vértices, que representam os pesquisadores que realizam pesquisa sobre coautoria e que tiveram publicações científicas indexadas na base WoS. 0 universo temporal é uma faixa de 16 anos e foram avaliadas a relação geográfica e as áreas de publicação. Os resultados são apresentados na seção 3 .

\subsection{Tratamento de dados da rede}

Quando se trabalha com análise de redes muitas vezes são necessários ajustes no universo pesquisado devido ao tamanho da rede ou de particularidades que podem impactar no entendimento da estrutura apresentada. Essas manipulações devem ser declaradas para evitar 
análises tendenciosas. O modelo exploratório proposto funciona bem com redes de pequeno ou médio porte, com até algumas centenas de vértices. Redes com dezenas de milhares de vértices geram dificuldade na inspeção visual.

$\mathrm{Na}$ base trabalhada, optou-se por excluir da rede os pesquisadores que possuíam apenas uma publicação na área. A escolha de reduzir o tamanho da rede se deu por acreditar-se que uma vez que o foco deste estudo é a colaboração científica, esta não está bem representada com a publicação de apenas um artigo. Os pesquisadores retirados do universo poderiam ter um número maior de publicações em outras áreas do conhecimento, mas como o recorte realizado teve como foco a área de coautoria, os pesquisadores com poucas publicações nessa área apresentam pouca relevância ao estudo.

\subsection{Determinação das característica estruturais}

Nas pesquisas de análise de redes, muitas características estruturais são quantificadas por meio de medidas, como, por exemplo, a centralidade ou a densidade. As medidas utilizadas no percurso deste trabalho estão descritas na Tabela 1 . Algumas medidas dizem respeito a toda a rede, e outras têm como foco os vértices ou as linhas. O estudo das características estruturais é muito mais preciso e conciso do que as análises visuais e serve como apoio para a inspeção visual.

Uma característica da rede, mas que também é utilizada no tratamento dos dados desta, é o chamado "componente gigante", que é o maior elemento da rede, onde os vértices daquele grupo se encontram todos conectados entre si. Quanto à estrutura, o estudo do componente gigante ajuda a identificar se a rede é bastante coesa ou esparsa e dividida em pequenos grupos. Estudos acerca de redes de colaboração científica revelam a tendência de colaboração intensa entre diferentes atores, o que torna o componente gigante o elemento de maior relevância na rede, uma vez que a maioria dos pesquisadores se encontra conectada entre si.

\subsection{Inspeção visual}

A visualização das redes ou inspeção visual é um elemento chave na análise exploratória, possibilitando o mapeamento e a apresentação dos padrões de relacionamentos. As redes com milhares de vértices prejudicam ou dificultam essa etapa do processo por causa das próprias limitações do olho humano.

Para evitar esse tipo de problema faz-se necessário uma série de precauções metodológicas visando garantir a melhor apresentação de resultados possível para que se consiga obter uma interpretação mais fidedigna.

A distribuição dos vértices na rede é um elemento essencial para essa análise onde são utilizados algoritmos de distribuição. Dois dos 
algoritmos utilizados neste trabalho foram o Fruchterman Reingold ${ }^{1}$ e o Force Atlas-2. ${ }^{2}$ o primeiro faz uma distribuição circular dos vértices, colocando os elementos com maior centralidade no centro da esfera. 0 segundo foca nos relacionamentos mais fortes e evidentes, demonstrando a coesão dos grupos. Além disso, para a definição de subgrupos dentro das redes foi utilizado um algoritmo de clusterização/agrupamento. Estes subgrupos foram identificados com cores diferentes para caracterizar a participação em um determinado cluster.

O tamanho dos vértices e a espessura das linhas de ligação entre os pesquisadores também foram utilizados para representar o número de elementos e ligações ou o grau de centralidade em alguns casos. Todas as características apresentadas nas figuras foram explicadas na sua interpretação.

\section{Dados bibliométrico}

O primeiro passo é a descrição bibliométrica do universo pesquisado, ou a "definição da rede", como é chamado na metodologia que está sendo utilizada. Para este estudo foi utilizada a base WoS, pois, além de possibilitar a análise das colaborações científicas também se pode realizar uma análise de citações, o que permite definir os artigos mais relevantes.

A pesquisa foi realizada em dezembro de 2014 e usou como termos de busca em tópicos "co-author*", "scientific collaboration" e "collaboration network". O caractere de truncagem "*" foi utilizado para abarcar todas as formas da palavra coautoria em inglês. Foi utilizado também o operador booleano "OR" entre os termos. A pesquisa resultou em 851 artigos entre o período de 2000 a 2014, com foco nas categorias Information Science and Library Science, Multidisciplinary Science, Multidisciplinary Physics, Mathematical Physics e Social Sciences Interdisciplinary. As categorias são uma classificação feita pela WoS que está relacionada diretamente às revistas nas quais os artigos são publicados.

A escolha por essas categorias foi feita com base em uma análise qualitativa dos artigos onde aqueles relacionados à Ciência da Informação e à Ciência de Redes com alto número de citações tiveram maior peso. Muitos artigos das categorias de ciência da computação foram encontrados, mas estes tinham como foco principal o estudo de métodos e algoritmos para a ciência de redes, e não o estudo de redes de colaboração. A Figura 1 mostra o crescimento do número de publicações ao longo dos últimos anos na pesquisa sobre colaboração científica.

\footnotetext{
${ }^{1}$ Disponível em: <https://github.com/gephi/gephi/wiki/Fruchterman-Reingold>. Acesso em: 15 jun. 2015.

${ }^{2}$ Disponível em: <https://github.com/gephi/gephi/wiki/Force-Atlas-2>. Acesso em: 15 jun. 2015.
} 
Figura 1 - Número de publicações sobre coautoria por ano

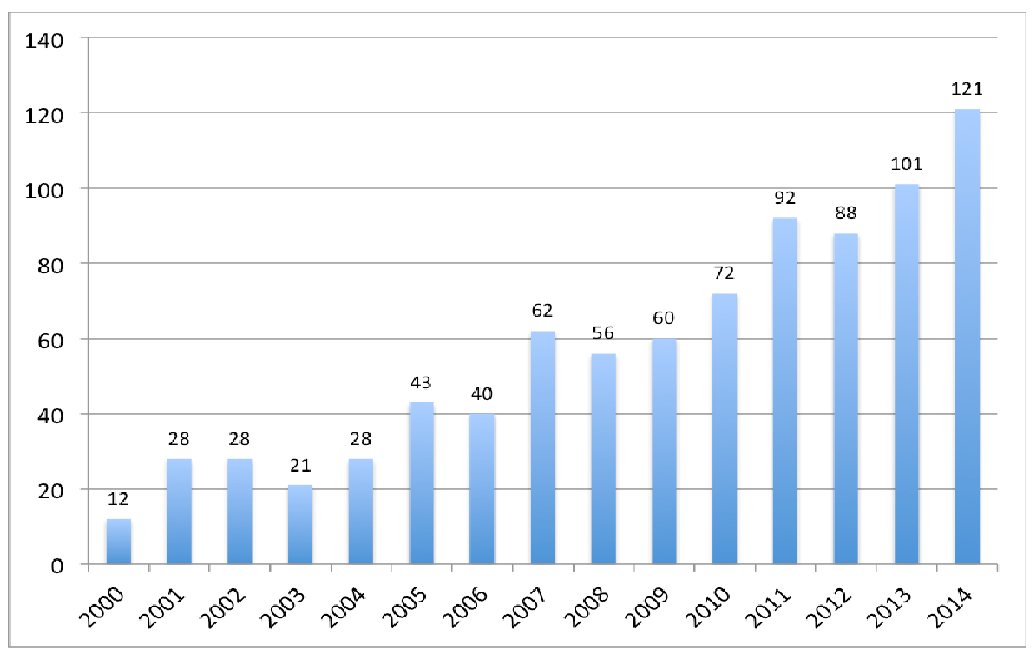

Fonte: Dados da pesquisa.

O que se percebe na Figura 1 é um crescimento constante das publicações nos últimos anos. No entanto, esse fator deve ser levado em consideração quanto ao número geral das publicações científicas, que também cresceu em todas as áreas da ciência. No caso das pesquisas em colaboração científica o aumento foi de 10 vezes o número de publicações comparando o ano de $2000 \mathrm{com}$ o de 2014. Essa evolução é elevada e relacionada diretamente à ampliação do interesse na área de ciência de redes, que começou seu grande boom a partir de trabalhos como os de Watts e Strogatz (1998), Barabási e Albert (1999) e Newman (2001). Outro fator que pode ter corroborado esse crescimento é a facilidade de acesso a novas tecnologias que otimizaram o tratamento de grandes bases de dados.

A Figura 2 apresenta o número de publicações por país. Os países com maior número de publicações são os Estados Unidos (EUA) e a China, seguidos de países europeus, com a Coreia do Sul na oitava posição e o Brasil na nona.

Figura 2 - Número de publicações por país

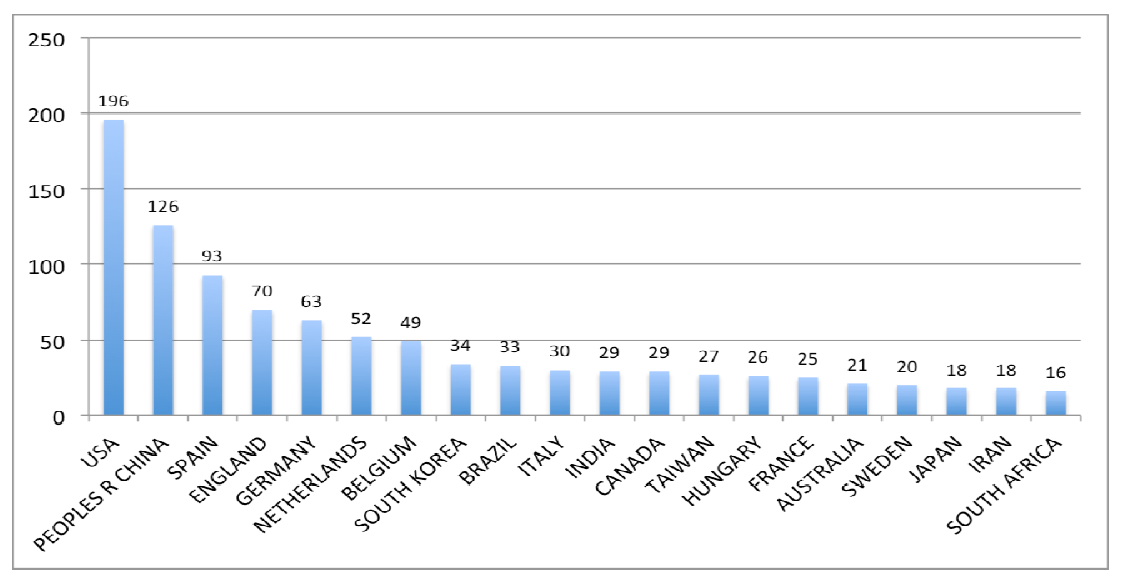

Fonte: Dados da pesquisa. período.

A Figura 3 apresenta os vinte pesquisadores que mais publicaram nesse 
Figura 3 - Número de publicações por autor

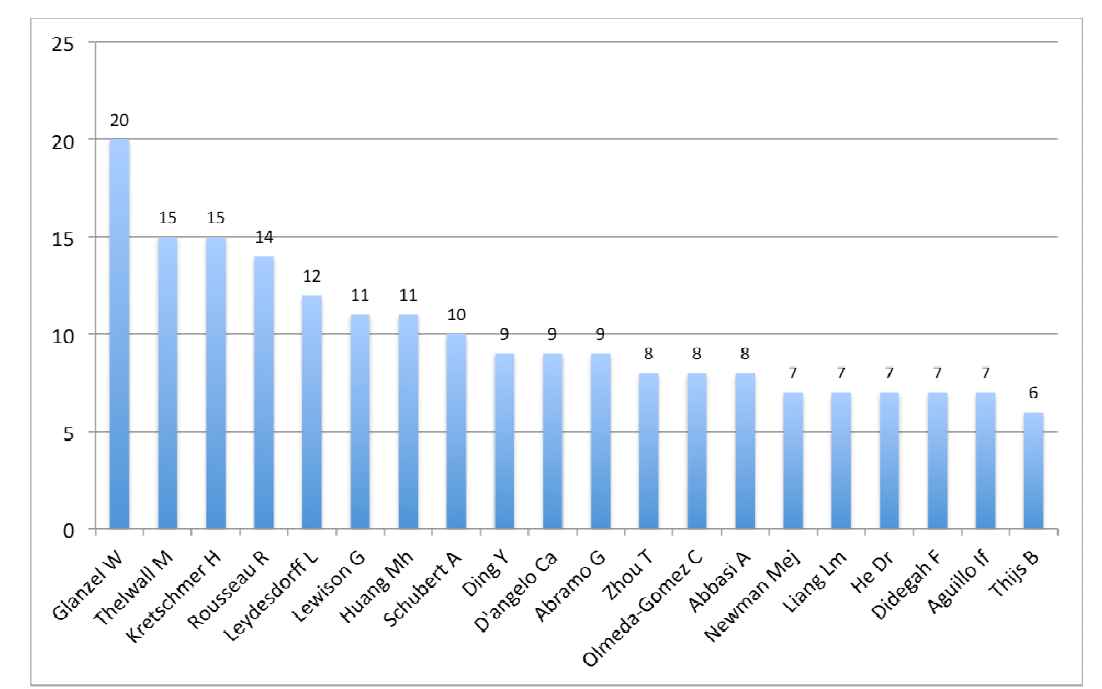

Fonte: Dados da pesquisa.

O pesquisador com maior número de publicações na área é Glänzel, com 20 publicações, sendo que algumas dessas apresentaram número elevado de citações. Apesar de a China ser o segundo país com maior número de publicações, seus pesquisadores não aparecem com tanto destaque, tendo quatro pesquisadores na lista dos 20 pesquisadores com maior número de publicações. Da lista de publicações por autor, dois autores destacam-se pela qualidade do trabalho quanto ao número de citações: Mark J. Newman e Loet Leydesdorff, sendo o primeiro físico e o segundo da área de ciência da informação. Esses pesquisadores, além de Glänzel, têm sido uma referência na área de redes de colaboração.

É importante ressaltar que existem pesquisadores renomados na ciência de redes, como, por exemplo, Barabási, que publicou sobre colaboração científica (BARABÁSI et al., 2002), mas o foco de seu trabalho tem sido outras áreas, incluindo a aplicação na saúde (BARABÁSI; OLTVAI, 2004; GOH et al., 2007), e por isso não aparece entre os que mais publicaram. Esse mesmo padrão ocorre no caso de outros pesquisadores com nível de citação muito acima da média, mas com baixo número de publicações.

\subsection{Rede de colaboração científica}

As características estruturais da rede podem ser observadas com 0 detalhamento dos elementos da rede apresentados na Tabela 1. Esses elementos fazem parte da descrição dos dados trabalhados e algumas medidas comuns ao estudo de redes. 
Tabela 1 - Medidas de Redes

\begin{tabular}{lc}
\hline Número de Vértices & 1705 \\
Número de Linhas & 4874 \\
Grau Médio & 5,717 \\
Número de Componentes & 402 \\
Vértices sem Colaborações & 107 \\
Componente Gigante & 169 \\
Diâmetro da Rede & 13 \\
Distância Média & 6,04 \\
\hline
\end{tabular}

Fonte: Dados da pesquisa.

Os dois primeiros elementos descrevem o número total de vértices e linhas da rede. A próxima medida é o grau médio da rede que se contabiliza como o número de colaborações de cada pesquisador somado e dividido pelo número total de vértices. Os componentes da rede são entendidos como todos os subgrupos separados que a rede apresenta. Quando um vértice aparece sem nenhuma colaboração, é considerado como mais um componente da rede. No caso do componente gigante, que representa o maior componente da rede com todos os seus pesquisadores conectados, este possui 169 vértices. O diâmetro da rede deve ser avaliado com base no componente gigante e calcula a maior distância entre dois vértices da rede. Por último é apresentado a distância média da rede que calcula a distância entre quaisquer dois vértices da rede em média.

Para a análise da rede de pesquisa sobre coautoria objetivamos focar na inspeção visual para apoiar a definição das características estruturais das redes e melhor entender os resultados. A Figura 4 apresenta a rede com distribuição Frunchterman Reingold e apenas com os vértices com duas ou mais ligações para melhor visualização do grafo.

O grafo ou sociograma apresentado na Figura 4 representa a rede de colaboração científica acerca de coautoria. No sociograma foram colocados em destaque por meio de cores os grupos nos quais vários pesquisadores estão conectados. O maior grupo de pesquisadores conectados é chamado de componente gigante e representa nesta rede apenas $9 \%$ dos pesquisadores. 
Figura 4 - Rede de colaboração em pesquisa sobre coautoria

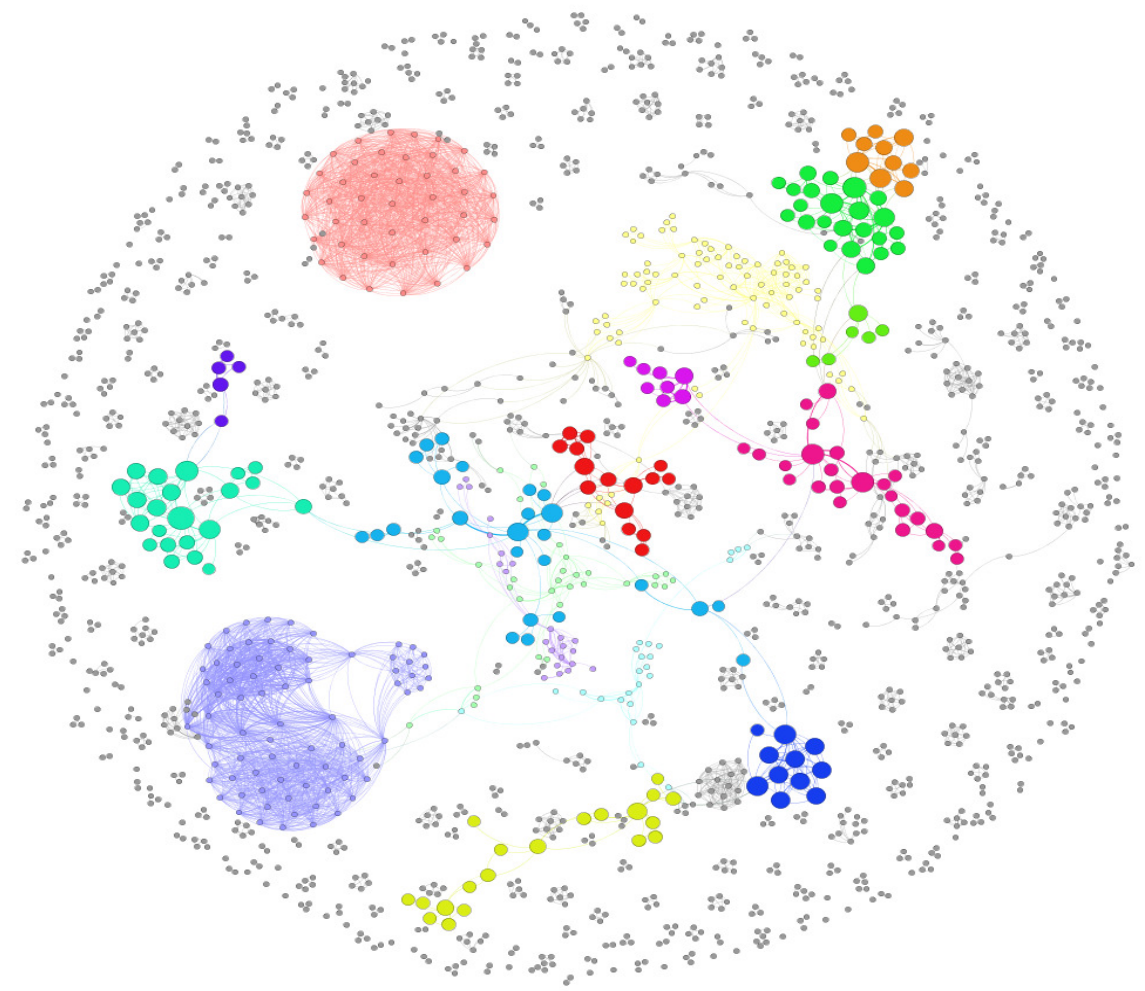

Fonte: Dados da pesquisa.

A Figura 4 representa uma rede de coautoria em que os vértices representam os pesquisadores e as linhas, as relações entre eles. Os vértices em cores fazem parte de grupos com número elevado de pesquisadores, enquanto os vértices em cinza fazem parte de grupos menores e não conectados aos demais pesquisadores. No centro da figura, os vértices com tamanho maior que os demais fazem parte de um mesmo grupo, apesar de apresentarem diferentes cores.

Essa estrutura bastante desconectada pode ser explicada pela característica transdisciplinar da área de pesquisa. Desse modo, entendemos que a análise de redes é um conjunto de métodos derivados da ciência de redes e utilizado por pesquisadores das mais diversas áreas. Mesmo se tratando do mesmo tema, que neste caso é a pesquisa a respeito da coautoria, os participantes desse grupo pertencem a diferentes áreas e têm pouca interação, como se percebe na Figura 4.

Dois grandes grupos bastante coesos são formados na parte superior e inferior esquerda, respectivamente. Esse tipo de formação ocorre devido à existência de artigos com um número muito elevado de pesquisadores; o grupo de cor avermelhada na parte superior tem apenas um artigo no qual todos colaboraram, e o de cor azulada tem poucos artigos.

O grupo de maior interesse ou foco de pesquisa é o que está ao centro, pois apesar de estarem todos conectados, ainda formam subgrupos dentro dessa própria rede. Os subgrupos são diferenciados 
pelas cores e foram definidos como pertencentes a este grupo por meio de um algoritmo de clusterização.

O que se pode perceber é que a área tem crescido bastante nos últimos anos, mas ainda não se consolidou como uma área específica de pesquisa na qual seus colaboradores trabalham em conjunto. Áreas de pesquisa já estabelecidas apresentam características de colaboração diferentes do que foi apresentado, lembrando que o recorte utilizado foi bastante específico para o universo que está em estudo neste trabalho. Por isso existe um número grande e crescente de pessoas na área, mas que não necessariamente colaboram entre si. Com o passar dos anos a tendência é que essa colaboração aumente por meio de participação em congressos, publicações, além da migração de pesquisadores para diferentes regiões, estabelecendo novos relacionamentos.

A Figura 5 apresenta com mais detalhes os pesquisadores que fazem parte do componente gigante ou do componente com o maior número de pesquisadores que colaboram entre si. Nesta figura buscou-se dar ênfase aos subgrupos, e com isso tentar entender onde estão os principais pesquisadores e como eles influenciam a formação do referido grupo.

Figura 5: Formação de Clusters no Componente Gigante

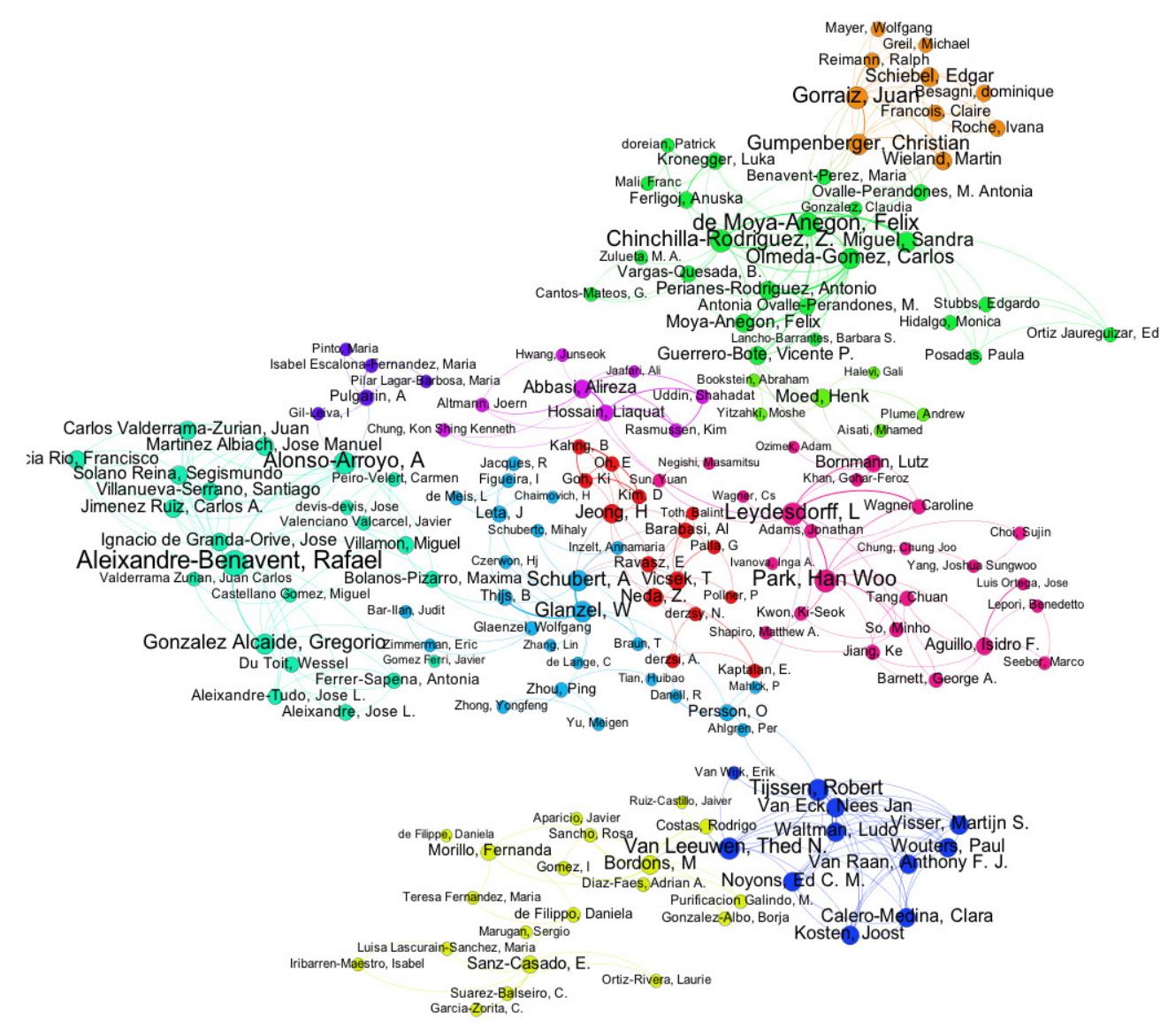

Fonte: Dados da pesquisa.

Os pesquisadores do componente gigante estão destacados por cores de acordo com o grupo definido por suas relações. O tamanho de 
cada nó varia de acordo com o número de colaborações que este possui. As linhas caracterizam as colaborações entre os pesquisadores.

No centro da Figura 5 percebem-se pesquisadores como Glänzel no grupo azul e Leydesdorf no grupo rosa, mais à direita da figura. Esses dois pesquisadores são apontados como sendo de alta relevância na área devido ao número de artigos, bem como artigos com alto número de citações.

Desse modo, percebe-se que, principalmente a inspeção visual das redes por meio de sociogramas favorece o entendimento do panorama das redes de colaboração dos diversos prismas especificados.

\section{Conclusão}

Sendo o objetivo desse trabalho, avaliar a capacidade de análise de resultados bibliométricos associados aos métodos de análise de redes, observou-se que tal associação contribui para resultados com melhor interpretação, principalmente por meio da inspeção visual que a análise de redes proporciona.

A análise da rede de pesquisadores que publicam sob o tema de coautoria não foi detalhada nesse trabalho, pois se buscou apenas analisar a capacidade de avaliação de resultados bibliométricos associados aos métodos de análise de redes. A interpretação das colaborações entre os diferentes autores e suas redes necessita de uma avaliação mais qualitativa, com a busca do entendimento acerca dos temas mais específicos, além de suas regiões ou grupos de pesquisa.

\section{Referências}

BARABÁSI, A.L.; ALBERT, R. Emergence of scaling in random networks. Science, Cambridge, v. 286, n. 5439, p. 509-512, 15 out. 1999.

BARABÁSI, A.L. Graph theory. Network Science. 2012. Disponível em: <http://barabasilab.neu.edu/networksciencebook/download/network_ science_November_Ch2_2012.pdf>. Acesso em: 22 maio 2014.

BARABÁSI, A.-L.; JEONG, H.; NEDA, Z. Evolution of the social network of scientific collaborations. Physica A: Statistical Mechanics and its Applications, v. 311, n. 3-4, p. 590-614. doi: 10.1016/S03784371(02)00736-7, 2002.

BARABÁSI, A.-L.; OLTVAI, Z. N. Network biology: understanding the cell's functional organization. Nature Reviews Genetics, v. 5, n. 2, p. 101-113. doi: 10.1038/nrg1272, 2004.

GLÄNZEL, W. Coathorship patterns and trends in the sciences (19801998): a bibliometric study with implications for database indexing and search strategies. Library Trends, v. 50, n. 3, p. 461, 2002.

GLÄNZEL, W.; SCHUBERT, A. Analyzing scientific networks through coauthorship. Nordic Journal Of Psychiatry, v. 57, n. 5, p. 393-394, 1 set. 2003. 
GOH, K.-Il et al. The human disease network. Proceedings of the National Academy of Sciences, v. 104, n. 21, p. 8685-90, 14 maio 2007.

GOYAL, S.; LEIJ, M. J. van D.; MORAGA-GONZÁLEZ, J. L. Economics: an emerging small world. Journal Of Political Economy, v. 114, n. 2, p. 403412, abr. 2006.

KATZ, J. S.; MARTIN, B. R. What is research collaboration? Research Policy, v. 26, n. 1, p.1-18, mar. 1997. Disponível em: <http://api.elsevier.com/content/article/PII:S0048733396009171 ?httpAcc ept=text/xml $>$. Acesso em: 23 out. 2015.

LAUDEL, G. What do we measure by co-authorships? Research Evaluation, v. 11, n. 1, p.3-15, 1 abr. 2002.

MELIN, G.; PERSSON, O. Studying research collaboration using coauthorships. Scientometrics, v. 36, n. 3, p. 363-377, jul. 1996.

NAGPAUL, P. S. Visualizing cooperation networks of elite institutions in India. Scientometrics, v. 54, n. 2, p. 213-228, 2002.

NATIONAL RESEARCH COUNCIL. Network Science. 1. ed. Washington, DC: National Academies Press, 2005.

NEWMAN, M. E. J. Networks: an introduction. USA: Oxford University Press, 2010.

NEWMAN, M. E. J. The structure of scientific collaboration networks. Proceedings Of The National Academy Of Sciences, v. 98, n. 2, p. 404-409, 16 jan. 2001. Disponível em: <http://www.pnas.org/content/98/2/404.full>. Acesso em: 10 out. 2014.

NEWMAN, M. E. J. Coauthorship networks and patterns of scientific collaboration. Proceedings of The National Academy of Sciences, v. 101, n. 1, p. 5200-05, 26 jan. 2004.

NOOY, W. de; MRVAR, A.; BATAGELJ, V. Exploratory social network analysis with Pajek. New York: Cambridge University Press, 2011.

SONNENWALD, D. H. Scientific collaboration. Annual Review Of Information Science And Technology, v. 41, n. 1, p. 643-681, 2007.

WASSERMAN, S.; FAUST, K. Social network analysis: methods and applications. Cambridge: Cambridge University Press, 1994.

WATTS, D. J.; STROGATZ, S. H. Collective dynamics of 'small-world' networks. Nature, v. 393, n. 6684, p. 440-442, 4 jun. 1998. 